\title{
Analysis of hydrodynamics and heat transfer in a thin liquid film flowing over a rotating disk by integral method
}

\author{
by \\ S. Basu and B. M. Cetegen* \\ Mechanical Engineering Department \\ University of Connecticut, Storrs, CT 06269-3139
}

\begin{abstract}
An integral analysis of hydrodynamics and heat transfer in a thin liquid film flowing over a rotating disk surface is presented for both constant temperature and constant heat flux boundary conditions. The model is found to capture the correct trends of the liquid film thickness variation over the disk surface and compare reasonably well with experimental results over the range of Reynolds and Rossby numbers covering both inertia and rotation dominated regimes. Nusselt number variation over the disk surface shows two types of behavior. At low rotation rates, the Nusselt number exhibits a radial decay with Nusselt number magnitudes increasing with higher inlet Reynolds number for both constant wall temperature and heat flux cases. At high rotation rates, the Nusselt number profiles exhibit a peak whose location advances radially outward with increasing film Reynolds number or inertia. The results also compare favorably with the full numerical simulation results from an earlier study as well as with the reported experimental results.
\end{abstract}

\section{Introduction}

Many investigations have been performed in the past on flow and heat transfer characteristics in thin liquid films due to the fact that high heat transfer rates can be obtained in thin films as reviewed by Webb and Ma [1]. Hydrodynamic characteristics of thin liquid films flowing over stationary and rotating disk surfaces are important in understanding the major factors affecting the heat transfer performance. A better

\footnotetext{
*Corresponding author, Phone (860)486-2966, E-mail cetegen@engr.uconn.edu
} 
understanding of the fluid mechanics of the liquid film flow allows better design and optimization of high performance compact heat transfer systems such as those employed in space applications, where considerations on efficiency, size, and weight are of critical importance. Study of rotating thin-film fluid physics and heat transfer is also of fundamental interest in the development of compact vapor absorption systems.

Watson [2] was first to analyze a free-falling jet impinging on a horizontal stationary plate. The film flow was divided into four regions. The first region was the impingement zone, followed by a second region of a growing boundary layer in the liquid film which eventually reaches to the film surface. Third region comprised of the transition to a fully developed film flow followed by a fully developed flow regime identified as the fourth region. For stationary and rotating liquid films at low flow rates and rotation speeds, a hydraulic jump could exist that has been studied both computationally and experimentally under normal and zero gravity conditions by Faghri and coworkers [3,4] and Avedisian and Zhao [5]. The hydraulic jump phenomenon was found to disappear at zero gravity conditions. Labus and Dewitt [6] conducted a combined numerical and experimental study to determine the free surface of a circular jet impinging on a flat plate in microgravity. The governing potential flow equations were solved numerically. The experimental study was performed using a drop-tower. The flow patterns of the free surface were examined experimentally, concluding that the surface tension and inertia were the dominant forces acting on the liquid. The experimental results were found to be in a good agreement with the numerical results. Thomas et al. [3] performed a useful one-dimensional analysis of the film thickness including the hydraulic jump phenomenon. They predicted significant thinning of the film with increased levels of rotation. They neglected inertia in their analysis.

Miyasaka [7] performed a similar theoretical and experimental study of the thickness of a thin viscous liquid film on a rotating disk. He obtained the film thickness by solving the governing equations of motion in the inviscid and viscous limits, with the latter utilizing the boundary layer approximation. Miyasaka also carried out experiments by using a liquid jet falling onto the center of a rotating disk. He deduced the film height from the electrical resistance of the liquid on the disk by comparing it to the electrical resistance of a standard thickness of the fluid. The computed values were found to agree 
with the experimental results. Rahman et al. [4] was the first to report a full numerical solution of the momentum equations using a finite difference scheme. The method utilized a boundary-fitted coordinate gridding scheme with a $k-\varepsilon$ model for turbulence closure and an iterative technique to define the free surface. They predicted the liquidfilm thickness in the vicinity hydraulic jump reasonably well and evaluated the effects at the outer edge of the disk. Rahman and Faghri [8] investigated the hydrodynamic behavior of a thin liquid film flowing over a rotating disk. They used a three-dimensional boundary-fitted coordinate system to perform the calculations. The computed film thickness agreed well with existing experimental measurements. It was also concluded that the flow was dominated by inertia near the entrance and by centrifugal force near the outer edge the disk. The hydrodynamic characteristics of a radially spreading liquid jet on a horizontal plate were also predicted numerically by Buyevich and Ustinov [9]. However they reported no comparison with other studies.

Rao and Arakeri [10] performed an analytical study of free liquid jets on surfaces including circular plates, cones, and spheres. They used a boundary layer approximation and a third order polynomial for the velocity profile. The equations were solved by the integral method. Their work however did not include heat transfer in the film. Nevertheless, they were the first to use the integral approach to predict relevant parameters like film thickness. They also did not present any experimental validation of their data.

Azuma and Hoshino [11] examined the laminar-turbulent transition, liquid-film thickness, velocity profile, stability, and wall pressure fluctuations of thin liquid films on a stationary horizontal disk. The laminar-turbulent transition was determined as a function of the nozzle inside diameter, the gap height, and the volumetric flow rate. The liquid-film thickness measurements were performed using a needle probe. More recently, Ozar et al. [12] measured the liquid film thickness by a laser light reflection technique with which the spatial distributions of film thickness were captured including the hydraulic jump.

Heat transfer in liquid films was first analyzed by Chaudury [13] who incorporated Watson's results into the heat transfer analysis. Wang et al. [14] developed a two-domain solution in which the heat transfer at the liquid film and the solid disk were 
treated separately and matched at the liquid-disk interface. Rahman and Faghri [8] used mixed numerical and analytical methods to predict the heat transfer. For developing flow and heat transfer, a three dimensional numerical model was utilized. Also, a two dimensional analytical solution was formulated for developing heat transfer and fully developed flow assuming solid body rotation. For the case of fully developed heat transfer and fluid flow, a closed form solution was developed. This solution predicted that the Nusselt number, based on film thickness, approached a constant value in the fully developed regime. Carper et al. [15] evaluated the convective heat transfer from a jet of cooling oil to an approximately isothermal rotating disk. Correlations were presented for the average Nusselt number as influenced by rotational Reynolds number, jet Reynolds number and radius of impingement. Carper et al. [16] later extended their study to include the effect of Prandtl numbers. Vader et al. [17] studied the effects of jet velocity and temperature on the heat transfer between a planar water jet and a stationary heated plate. They concluded that the heat transfer performance was affected by the free stream turbulence intensity and the Prandtl number. A similar study was presented by Stevens and Webb [18] where the influence of jet Reynolds number, nozzle to plate, spacing and jet diameter were evaluated. Empirical correlations were developed for stagnation point, local and average Nusselt numbers. Faghri et al. [19] presented heat transfer results for a controlled liquid impinging jet on a stationary disk. They presented a numerical study showing good agreement between heat transfer predictions and experimental data. Auone and Ramshaw [20] performed heat and mass transfer experiments on a liquid flowing over a rotating disk. They predicted the heat transfer coefficients analytically by adapting the solution that Nusselt [21] used for the film condensation under the influence of gravity. Ozar et al. [22] published an experimental study of heat transfer and reported the local and disk surface averaged Nusselt number over a range of flow rates and rotation speeds for water. Recently, Rice et al. [23] published full numerical computation of heat transfer in a thin liquid film over a rotating disk simulating the experimental conditions of Ozar et al. [22].

Review of the literature indicates that the analysis of the problem has progressed along two main themes. One theme has been numerical solution of the full problem taking into account its salient features. The other is of analytical nature utilizing 
simplifying assumptions to obtain simple analytical results. In between lies the studies that utilize the Karman-Pohlhausen type integral analyses. This type of analysis is capable of capturing sufficient details of the solution while avoiding oversimplifications needed in analytical studies. For example, many of the analytical analyses had not considered the liquid film inertia effects in the presence of rotation. It was thus the objective of this study to present a comprehensive integral analysis of the thin liquid film flowing radially outward on a circular disk including effects of inertia and rotation. Capability of the integral method to predict the flow and heat transfer is demonstrated by comparisons with experimental and numerical results for the same problem.

\section{Problem Formulation}

The rotating disk is schematically shown in Fig. 1 which resembles the experimental set-up that has been utilized by Thomas et al [3] and Ozar et al [12,22] in a series of experimental studies. In the experiments, the flow is introduced from a central collar that directs the liquid radially outward over a gap height of $h_{o}$. The liquid flows over the rotating disk while being heated from underneath by an electric resistance heater. In these experimental studies, the liquid film thickness and heat transfer coefficients were measured. Liquid film thickness measurements were made by either a capacitance probe [3] or an optical technique [12]. The heat transfer coefficients were determined from the difference between the measured disk surface temperature and the liquid inlet temperatures and the constant heat flux supplied to the disk.

The problem is considered in the radial $(r)$ and axial $(z)$ coordinates assuming azimuthal symmetry. For this situation, the governing equations in cylindrical coordinate system over a rotating circular disk are:

$$
\begin{array}{ll}
\text { Continuity: } & \frac{1}{r} \frac{\partial}{\partial r}\left(r u_{r}\right)+\frac{\partial u_{z}}{\partial z}=0 \\
\text { r-momentum: } & \frac{1}{r} \frac{\partial}{\partial r}\left(r u_{r} u_{r}\right)+\frac{\partial\left(u_{r} u_{z}\right)}{\partial z}=r \omega^{2}+v\left[\frac{\partial^{2} u_{r}}{\partial z^{2}}-\frac{u_{r}}{r^{2}}+\frac{1}{r} \frac{\partial}{\partial r}\left(r \frac{\partial u_{r}}{\partial r}\right)\right] \\
\text { Energy: } & \frac{1}{r} \frac{\partial}{\partial r}\left[r u_{r} T\right]+\frac{\partial}{\partial z}\left[u_{z} T\right]=\alpha\left[\frac{1}{r} \frac{\partial}{\partial r}\left(r \frac{\partial T}{\partial r}\right)+\frac{\partial^{2} T}{\partial z^{2}}\right]
\end{array}
$$

Defining non-dimensional parameters as, 


$$
\tilde{r} \equiv \frac{r}{r_{0}}, \tilde{u}_{r} \equiv \frac{u_{r}}{u_{o}}, \tilde{z} \equiv \frac{z}{h_{o}}, \tilde{u}_{z} \equiv \frac{u_{z}}{u_{o}}, \theta_{T} \equiv \frac{T-T_{o}}{T_{o}-T_{i}}, \theta_{q} \equiv\left(\frac{k}{q_{o} h_{o}}\right)\left(T-T_{i}\right),
$$

where $r_{0}$ is the inlet radius of the disk, $u_{0}$ is the inlet velocity of the liquid jet, $h_{0}$ is the collar height, $\theta_{T}$ is the nondimensional temperature for the constant wall temperature and $\theta_{q}$ is its counterpart for constant wall heat flux. $T_{0}$ is the temperature of the disk surface for constant wall temperature case, $T_{i}$ is the inlet temperature of the liquid jet, $q_{0}$ is the heat flux supplied to the disk for the constant wall heat flux case. Non-dimensionalizing the governing equations we obtain,

$$
\begin{aligned}
& \frac{1}{\tilde{r}} \frac{\partial}{\partial \tilde{r}}\left(\tilde{r} \tilde{u}_{r}\right)+\left(\frac{r_{o}}{h_{o}}\right) \frac{\partial \tilde{u}_{z}}{\partial \tilde{z}}=0 \\
& \frac{1}{\tilde{r}} \frac{\partial}{\partial \tilde{r}}\left[\tilde{r} \tilde{u}_{r}^{2}\right]+\left(\frac{r_{o}}{h_{o}}\right) \frac{\partial}{\partial \tilde{z}}\left[\tilde{u}_{r} \tilde{u}_{z}\right]=\frac{\tilde{r}}{R o}+\frac{1}{\operatorname{Re}}\left[\frac{1}{\tilde{r}} \frac{\partial}{\partial \tilde{r}}\left(\tilde{r} \frac{\partial \tilde{u}_{r}}{\partial \tilde{r}}\right)+\left(\frac{r_{o}}{h_{o}}\right)^{2} \frac{\partial^{2} \tilde{u}_{r}}{\partial \tilde{z}^{2}}-\frac{\tilde{u}_{r}}{\tilde{r}^{2}}\right] \\
& \frac{1}{\tilde{r}} \frac{\partial}{\partial \tilde{r}}\left[\tilde{r} \tilde{u}_{r} \theta\right]+\left(\frac{r_{o}}{h_{o}}\right) \frac{\partial}{\partial z}\left[\tilde{u}_{z} \theta\right]=\frac{1}{P e}\left\lceil\frac{1}{\tilde{r}} \frac{\partial}{\partial \tilde{r}}\left(\tilde{r} \frac{\partial \theta}{\partial \tilde{r}}\right)+\left(\frac{r_{o}}{h_{o}}\right)^{2} \frac{\partial^{2} \theta}{\partial \tilde{z}^{2}}\right]
\end{aligned}
$$

where Reynolds, Rossby and Peclet numbers are defined as

$$
\operatorname{Re} \equiv \frac{u_{o} r_{o}}{v}, R o \equiv \frac{u_{o}^{2}}{\omega^{2} r_{o}^{2}}, P e \equiv \frac{u_{o} r_{o}}{\alpha}
$$

where $v$ is the kinematic viscosity and $\alpha$ is the thermal diffusivity, both of which are assumed to be constant in the context of this analysis.

Since $r_{o}>h_{o}$, then, $\frac{r_{o}^{2}}{h_{o}^{2}}>>1$, we can infer that

$$
\left(\frac{r_{o}}{h_{o}}\right)^{2} \frac{\partial^{2} \tilde{u}_{r}}{\partial \tilde{z}^{2}} \gg \frac{1}{\tilde{r}} \frac{\partial}{\partial \tilde{r}}\left(\tilde{r} \frac{\partial \tilde{u}_{r}}{\partial \tilde{r}}\right) \text { and }\left(\frac{r_{o}}{h_{o}}\right)^{2} \frac{\partial^{2} \tilde{u}_{r}}{\partial \tilde{z}^{2}} \gg>\frac{\tilde{u}_{r}}{\tilde{r}^{2}} \text { and }\left(\frac{r_{o}}{h_{o}}\right)^{2} \frac{\partial^{2} \theta}{\partial \tilde{z}^{2}} \gg>\frac{1}{\tilde{r}} \frac{\partial}{\partial \tilde{r}}\left(\tilde{r} \frac{\partial \theta}{\partial \tilde{r}}\right)
$$

Hence the momentum and energy equations take the form

$$
\begin{aligned}
& \frac{1}{\tilde{r}} \frac{\partial}{\partial \tilde{r}}\left[\tilde{r} \tilde{u}_{r}^{2}\right]+\left(\frac{r_{o}}{h_{o}}\right) \frac{\partial}{\partial \tilde{z}}\left[\tilde{u}_{r} \tilde{u}_{z}\right]=\frac{\tilde{r}}{R o}+\frac{1}{\operatorname{Re}}\left[\left(\frac{r_{o}}{h_{o}}\right)^{2} \frac{\partial^{2} \tilde{u}_{r}}{\partial \tilde{z}^{2}}\right] \\
& \frac{1}{\tilde{r}} \frac{\partial}{\partial \tilde{r}}\left[\tilde{r} \tilde{u}_{r} \theta\right]+\left(\frac{r_{o}}{h_{o}}\right) \frac{\partial}{\partial \tilde{z}}\left[\tilde{u}_{z} \theta\right]=\frac{1}{P e}\left(\frac{r_{o}}{h_{o}}\right)^{2} \frac{\partial^{2} \theta}{\partial \tilde{z}^{2}}
\end{aligned}
$$




\section{Liquid Film Hydrodynamics}

Integrating the momentum equation with respect to $\tilde{z}$ from 0 to $\tilde{\delta}=\frac{\delta}{h_{o}}$, we get,

$$
\frac{1}{\tilde{r}} \frac{\partial}{\partial \tilde{r}} \int_{0}^{\bar{\delta}} \tilde{r} \tilde{u}_{r}^{2} d \tilde{z}+\frac{r_{o}}{h_{o}}\left[\tilde{u}_{r} \tilde{u}_{z}\right]_{\tilde{\delta}}=\frac{\tilde{r} \tilde{\delta}}{\operatorname{Ro}}-\frac{1}{\operatorname{Re}}\left[\left.\left(\frac{r_{o}}{h_{o}}\right)^{2} \frac{\partial \tilde{u}_{r}}{\partial \tilde{z}}\right|_{0}\right]
$$

From continuity equation, we can write

$$
\tilde{u}_{z}(\tilde{\delta})=-\frac{h_{o}}{r_{o} \tilde{r}} \frac{\partial}{\partial \tilde{r}} \int_{0}^{\delta} \tilde{r}_{r} d \tilde{z}
$$

A parabolic radial velocity profile is assumed as,

$$
\tilde{u}_{r}=a_{0}+a_{1} \tilde{z}+a_{2} \tilde{z}^{2}
$$

subject to the boundary conditions of no slip at the wall $\tilde{u}_{r}(\tilde{z}=0)=0$ and no shear at the free surface $\frac{\partial \tilde{u}_{r}}{\partial \tilde{z}}(\tilde{z}=\tilde{\delta})=0$. In addition, the total volume flow at any cross-section perpendicular to $r$ has to be equal to the inlet flow rate for the case of no vaporization or mass loss, these conditions allow determination of coefficients in the velocity profile leading to,

$$
\tilde{u}_{r}=3\left(\frac{\tilde{z}}{\tilde{r} \tilde{\delta}^{2}}\right)-\frac{3}{2}\left(\frac{\tilde{z}^{2}}{\tilde{r} \tilde{\delta}^{3}}\right)
$$

Substituting this profile into the integral momentum equation and integrating with respect to $z$, one gets

$$
\frac{d \tilde{\delta}}{d \tilde{r}}+\frac{\tilde{\delta}}{\tilde{r}}+\frac{5}{6 \operatorname{Ro}} \tilde{r}^{3} \tilde{\delta}^{3}=\frac{5}{2 \operatorname{Re}}\left(\frac{r_{o}}{h_{o}}\right)^{2} \tilde{r}
$$

In this equation, the first two terms are due to advection, the third term is a result of rotation and the term on the right side represents the viscous shear. Equation 13 subject to the initial condition $\tilde{\delta}(\tilde{r}=1)=1$ was numerically integrated to determine the film thickness over the disk surface. Equation (13) becomes an algebraic equation for the case of negligible inertia in which case the first two terms disappear. The film thickness can be written in exact form as,

$$
\tilde{\delta}=\left[\frac{3 \operatorname{Ro}}{\operatorname{Re}}\left(\frac{r_{o}}{h_{o}}\right)^{2}\right]^{1 / 3} \frac{1}{\tilde{r}^{2 / 3}}
$$


This result is identical to that obtained based on the falling film analysis with gravity being replaced by the centrifugal force. Having the liquid film hydrodynamics established, we now proceed with the heat transfer analysis in the film.

\section{Heat Transfer in the Liquid Film}

The heat transfer in the liquid film is analyzed by considering the two cases of constant disk surface temperature and constant disk surface heat flux. In either case, there is a thermal entry region where the thermal boundary layer lies below the film surface as shown in Fig. 1. As it is shown in Appendix A for constant disk surface temperature case, the thermal entry region length scales as,

$$
\tilde{r}_{*}=\left[1+\frac{P e}{6}\left(\frac{h_{o}}{r_{o}}\right)^{2}\right]^{1 / 2}
$$

For moderate Peclet numbers, $\tilde{r}_{*} \approx 1\left(o r r_{*} \approx r_{o}\right)$ since $h_{o}<<r_{o}$, thus allowing us to neglect the entry length.

\section{Constant disk surface temperature case}

For this case, the temperature profile can be constructed as a second order polynomial given by,

$$
\theta=a_{0}+a_{1}\left(\frac{z}{\delta_{r}}\right)+a_{2}\left(\frac{z}{\delta_{T}}\right)^{2}
$$

which has to satisfy the boundary conditions: $\theta=0$ at $z=0, \frac{d \theta}{d z}=0$ at $z=\delta_{T}$. The profile satisfying these conditions is,

$$
\theta=\theta_{\infty}\left[\left(\frac{z}{\delta_{T}}\right)^{2}-2\left(\frac{z}{\delta_{T}}\right)\right] \quad \text { where } \quad \begin{aligned}
& \delta_{T}<\delta \Rightarrow \theta_{\infty}=1 \\
& \delta_{T}=\delta \Rightarrow \theta_{\infty}=\frac{T_{\delta}(r)-T_{o}}{T_{o}-T_{i}}
\end{aligned}
$$

Substituting this profile along with the velocity profile into the integral energy equation, one obtains,

$$
\frac{d \theta_{\infty}}{d \tilde{r}}=-\theta_{\infty}\left[\frac{5}{2 P e}\left(\frac{r_{o}}{h_{o}}\right)^{2} \frac{\tilde{r}}{\tilde{\delta}}-\frac{5}{4} \frac{d \tilde{\delta}}{d \tilde{r}}\right]
$$

Integrating and applying the condition that $\theta_{\infty}\left(\tilde{r}=\tilde{r}_{*}\right)=1$, we get 


$$
\theta_{\infty}=\exp \left[-\frac{5}{2}\left(\frac{r_{o}}{h_{o}}\right)^{2} \frac{1}{P e} \int_{r_{*}}^{\tilde{r}} \frac{\tilde{r} d \tilde{r}}{\tilde{\delta}}+\frac{5}{4}\left(\tilde{\delta}-\tilde{\delta}_{*}\right)\right]
$$

Defining the Nusselt number as, $N u_{T} \equiv \frac{h r_{0}}{k}=-\left.\frac{r_{o}}{\tilde{\delta}} \frac{d \theta}{d \tilde{z}}\right|_{t=0}$

$$
N u_{T}=2 \frac{r_{o}}{h_{o} \widetilde{\delta}} \exp \left[-\frac{5}{2}\left(\frac{r_{0}}{h_{0}}\right)^{2} \frac{1}{P_{e}} \int_{r_{*}}^{\tilde{r}} \frac{\tilde{r} d \tilde{r}}{\widetilde{\delta}}+\frac{5}{4}\left(\tilde{\delta}-\tilde{\delta_{*}}\right)\right]
$$

The area-averaged Nusselt number can be defined as

$$
N u_{\text {avg }}=\frac{1}{\pi\left(\tilde{r}^{2}-1\right)} \int_{1}^{\tilde{r}} N u_{T} 2 \pi \tilde{r} d \tilde{r}
$$

\section{Constant heat flux case:}

The temperature profile for the constant heat flux case is subject to $\left.\frac{d \theta_{q}}{d \tilde{z}}\right|_{z=0}=-1$ and $\left.\frac{d \theta_{q}}{d \tilde{z}}\right|_{z=\delta}=0$, i.e., prescribed heat flux at the wall, $q_{o}$ and adiabatic free surface. With these conditions, the temperature profile becomes,

$$
\theta=a_{o}+\tilde{\delta}\left(\frac{\hat{z}^{2}}{2}-\hat{z}\right)
$$

where $a_{0}$ is a parameter which is a function of $\mathrm{r}$. Substituting this temperature profile into the integral form of the energy equation and integrating, one gets,

$$
\frac{d a_{0}}{d \tilde{r}}+a_{0} \frac{d \tilde{\delta}}{d \tilde{r}}=\frac{\tilde{r}}{P e}\left(\frac{r_{0}}{h_{0}}\right)^{2}+\frac{2}{5} \frac{d \tilde{\delta}}{d \tilde{r}}+\frac{\tilde{\delta}}{2} \frac{d \tilde{\delta}}{d \tilde{r}}
$$

subject to the initial value of $a_{0}(\tilde{r}=1)$. While this value can be taken as $a_{o}(\tilde{r})=1$ based on $\theta_{q}(\tilde{r}=1)=1$ in eqn. (22), a better estimate is provided by integrating the energy flux at $\tilde{r}=1, \int_{0}^{1}\left[\frac{q_{o} h_{o} \theta_{q}}{k T_{i}}+1\right] d \tilde{z}=1$, yielding $a_{o}(\tilde{r}=1)=1 / 3$. For the case of negligible inertia, an analytical solution for $a_{o}$ can be obtained as described in Appendix B,

$$
a_{o}=\left(\frac{r_{o}}{h_{o}}\right)^{2} \frac{\tilde{r}-1}{\operatorname{RePr}}-\frac{7}{20}\left(\frac{r_{o}}{h_{o}}\right)^{2 / 3}\left(\frac{3 R o}{\operatorname{Re}}\right)^{1 / 3}\left(\tilde{r}^{-2 / 3}-1\right)+\frac{1}{3}
$$

Nusselt number for the case of constant disk surface heat flux can be found from, 


$$
N u_{q} \equiv \frac{h r_{o}}{k}=\frac{q_{o} r_{o}}{k\left[T(\tilde{z}=0)-T_{i}\right]}=\frac{r_{o}}{a_{o} h_{o}}
$$

For the case of negligible inertia,

$$
N u_{q}=\left[\left(\frac{r_{o}}{h_{o}}\right) \frac{\tilde{r}-1}{\operatorname{RePr}}-\frac{3^{i / 3} 7}{20}\left(\frac{r_{o}}{h_{o}}\right)^{-1 / 3}\left(\frac{\operatorname{Ro}}{\operatorname{Re}}\right)^{1 / 3}\left(\tilde{r}^{-2 / 3}-1\right)+\frac{1}{3}\left(\frac{h_{o}}{r_{o}}\right)\right]^{T^{-1}}
$$

The averaged Nusselt number is obtained by integrating the local Nusselt number over the disk surface based on eqn. (21). In the following, the results obtained from this analysis are presented and discussed.

\section{Results and Discussion}

The calculation of the liquid film thickness and Nusselt numbers for constant wall temperature and constant heat flux cases were performed for a range of inlet Reynolds numbers $\operatorname{Re} \equiv \frac{u_{o} r_{o}}{v}$ and Rossby numbers $R o \equiv \frac{u_{o}^{2}}{\omega^{2} r_{o}^{2}}$. The range of parameters were chosen to cover those reported in the experiments of Ozar et al $[12,22]$ and the recent numerical simulations of Rice et al [23]. The value of the geometric parameter $\frac{r_{o}}{h_{o}}$ was taken to be same as in the experiments with a value of 200 . In the following, the computed results are presented and compared with numerical and experimental results mentioned above.

Figure 2 shows the variation of the film thickness over the disk surface with Reynolds number for two values of Rossby number. At low rotation speeds (Ro $=1000$ ), the film thickness initially increases along the disk radius and then begins to thin out at outer radii due to effect of rotation as seen in Fig. 2a. The film thickness decreases with increasing inlet velocity or Reynolds number and the peak film thickness location shifts to larger radii with increasing Re. The analytical result given by eqn. (14) is also shown in Fig. $2 \mathrm{a}$ for $\mathrm{Re}=10^{4}$. It is seen that the two results agree at large radii where the inertial effects are diminished and the negligible inertia result agrees with the full solution. At high rotation rates $(\mathrm{Ro}=0.5)$, film thickness exhibits a radial decay with the film thickness still decreasing with increasing inertia as shown in Fig. 2b. The variation 
of the film thickness for high levels of rotation is well represented by eqn. (14) as seen by the agreement at $\operatorname{Re}=10^{4}$. The effect of varying the rotation speed on the film thickness at a constant value of inlet velocity or Reynolds number is depicted in Fig. 3. With increasing rotation speed (i.e. decreasing Ro), the film thickness decreases. The trend at high Ro, which exhibits a maximum in the film thickness, gradually changes to a radially decaying film thickness at high rotation speeds (i.e. low Ro). This change appears to happen at around Ro $=10$ for this case. The computed results are in reasonably good agreement with the experimental results reported by Ozar et al [22] as shown in Fig. 4. The integral model captures the trend of liquid film thickness variation at different Reynolds and Rossby numbers. Due to the uncertainties in the experimentally measured film thickness, a closer agreement can not be expected.

Figure 5 shows the variation of the Nusselt number over the disk surface for the case of constant wall temperature. At low rotation rates $\left(\mathrm{Ro}_{10} 1000\right)$, shown in Fig. 5a, the Nusselt number decreases continuously with increasing radial distance. The magnitude of Nusselt number is highest at the entrance since the temperature gradient, and heat flux is the highest there at the liquid-disk interface. With increasing radial distance, the liquid film temperature increases and consequently the heat flux diminishes leading to a decrease in the heat transfer coefficient and Nusselt number. The value of Nusselt number approaches a constant value at large radii. Nusselt number increases with increasing inlet velocity or Reynolds number as expected. At high rotation speeds, shown in Fig. 5b, the trend of Nusselt number is quite different in that it increases from the inlet to a maximum value and decreases from that point on. The magnitude of Nusselt number increases with increasing Reynolds number with the peak Nusselt number location shifting radially outward. This prounounced increase in Nusselt number is due to the thinning of the liquid film due to inertia and rotation. The decrease at larger radii is due to the effect of heating of the liquid film reducing the temperature gradient and heat flux. At high rotation rates, Nusselt number increases significantly above the values for low rotation rates suggesting the strong enhancement of heat transfer due to rotation.

To better visualize the effects of rotation at a fixed value of inlet velocity or Reynolds number, Figure 6 shows the effect of Rossby number on Nusselt number 
variation. At low rotation speeds (i.e. high Ro), the Nusselt number is highest at the entrance and decreases with increasing radial distance. At about $\mathrm{Ro}=1$, the trend exhibits an increase of Nusselt number from the inlet reaching a maximum value followed by a decay. At higher rotation rates $\left(\mathrm{Ro}_{0}=0.5\right)$, this trend of Nusselt number becomes more pronounced with the maximum values of Nusselt number being an order of magnitude higher. The peak Nusselt number location shifts to smaller radii indicating that the rotational effects are felt at smaller radii with increasing rotation speed.

In Figure 7, the results are shown for the constant heat flux case at two values of Rossby number. At low rotation rates (i.e. high Ro), the Nusselt number variation is similar to that of constant wall temperature case (shown in Fig. 5a) except that the Nusselt number magnitude is higher than that for the constant wall temperature. The increase in Nusselt number is expected since the heat flux at the disk surface is maintained in this case. For the high rotation speed $\left(\mathrm{Ro}_{0}=0.5\right)$, Nusselt number exhibits a decay with increasing radial distance at low Reynolds number as the temperature of the liquid at the disk surface increases with increasing radial distance, similar to the constant wall temperature case. With increasing Reynolds number, the Nusselt number magnitude increases and it exhibits a maximum.

Figure 8 illustrates the influence of rotation on the Nusselt number variation with at a fixed value of inlet velocity or Reynolds number. It is found that rotation enhances the heat transfer into the liquid film very significantly. As the rotation rate reduces, the maximum in the Nusselt number variation disappears and it decays continuously with increasing radial distance. The location of the peak shifts to smaller radii with increasing rotation rate (i.e. decreasing $\mathrm{Ro}$ ) since the effects of rotation are felt further in with increasing rotation.

Figure 9 shows the comparison of the integral method results with the full numerical simulation results of Rice et al [23]. The comparisons are shown for $\mathrm{Re}=1.42$ $10^{4}$ and $2.8410^{4}$ for two rotation speeds of 50 and $100 \mathrm{rpm}$. The agreement between the integral model and numerical simulations appears to be good with maximum deviations of about $20 \%$. It is conceivable that the differences are due to variable fluid properties not accounted for in the integral model as well as the more detailed treatment afforded in the numerical model, such as conjugate heat transfer, evaporation effects etc. Figure 10 
displays the comparison of experimental results with the integral model for two cases. It is found that the experimental results good agreement with the model for these two cases with the radial variation of experimental Nusselt number being slightly less. Considering the uncertainty of the experimental data and the simplifications employed in the model, the level of agreement is remarkable.

The area averaged Nusselt numbers over the disk surface are shown in Figs. 11 and 12 respectively for the cases of constant wall temperature and constant heat flux. The trend of average Nusselt number is approximately linear for both cases with slightly decreasing curvature for the constant wall heat flux at low rotation rates (i.e. high Ro). The influence of Rossby number is nonlinear however and the average Nusselt number is found to scale as $\overline{N u} \propto R o^{-m}$ where $m \approx 0.55$ for constant wall temperature and $m \approx 0.18$ for constant heat flux. The stronger dependence for constant wall temperature can be rationalized based on the fact that heat transfer into the film is enhanced more for the constant wall temperature case in contrast to the constant wall heat flux.

Finally, Figure 13 shows the comparison of the analytical solution given by eqn. (26) for the negligible inertia case with the full solution for $\operatorname{Re}=10^{4}$ and $R o=0.5$. It is seen that the two solutions differ substantially near the entrance region, but merge at large radii where the inertial effects have diminished.

\section{Concluding Remarks}

A detailed integral analysis of flow and heat transfer in a thin liquid film flowing over a rotating disk was formulated to determine the liquid film thickness and Nusselt numbers for both constant wall temperature and heat flux cases. The results are presented for a range of inlet liquid flow rates or Reynolds numbers and Rossby numbers. It is found that the integral model captures the variation of film thickness over the disk radius for a range of parameters representing both inertia and rotation dominated regimes. At low rotation rates corresponding to high Rossby numbers, the film thickness grows radially until the centrifugal effect becomes sufficiently strong at large radii. For high inlet flow rates or Reynolds numbers, the film thickness decreases radially with decay being stronger with increasing inertia. For low inertia, the film thickness increases radially due to significant retardation of the film flow by viscosity. In cases where both 
inertial and rotational effects come into play, the film thickness first increases reaching a maximum followed by radial decay. The location of maximum film thickness changes based on the values of Reynolds and Rossby numbers. For cases dominated either by high rotation or low inertia, the film thickness is well represented by the analytical expression obtained for negligible inertia.

The analysis of the heat transfer in the liquid film indicate that the Nusselt number exhibits a radial decay at low rotation rates (i.e. high $\mathrm{Ro}$ ) for both cases of constant wall temperature and constant heat flux. The magnitude of Nusselt number is linearly dependent on inlet Reynolds number for both cases. However, the Nusselt number values for constant wall heat flux are greater than those for the constant wall temperature. At high rotation speeds and inlet Reynolds numbers, the Nusselt number first increases, reaches a peak and then displays a radial decay. The radial location of the peak Nusselt number shifts to larger radii with increasing Reynolds number and this is due to the competing effects of inertia and rotation on the film thickness and heat transfer characteristics. Effect of Rossby number appears to be significantly non-linear and high rotation rates can produce significant enhancement in heat transfer for both cases of constant wall temperature and heat flux. In addition to local Nusselt number variation, the disk surface area averaged Nusselt numbers were computed for both cases. They also show an approximately linear variation with respect to inlet flow rate or Reynolds number and inverse power law dependence on Rossby number. Rossby number dependence is stronger for the constant wall temperature case.

The results obtained from this integral analysis were compared with those from a recent numerical study of Rice et al [23] as well as the experiments of Ozar et al [21,22]. Both comparisons indicate good agreement with the integral model. Finally, the integral analysis provided analytical and semi-analytical expressions for the local Nusselt number in the limit of negligible inertia for the cases of constant wall heat flux and constant wall temperature respectively. 


\section{Acknowledgments}

The work presented in this article was funded by NASA Microgravity Fluid Physics

Program under Grant No. NCC3-789 with Dr. S. Sankaran as the grant monitor.

\section{References}

1. Webb, B. W. and Ma, C. F., 1995, "Single phase liquid impingement heat transfer," Adv. Heat Transfer, 26, pp. 105-217.

2. Watson, E. J.,1964, "The radial spread of a liquid jet over a horizontal plane," $J$. Fluid Mech., 20, pp. 481-499

3. Thomas, S., Hankey, W., Faghri, A., Swanson, T. ,1990, "One-dimensional analysis of the hydrodynamic and thermal characteristics of thin film flows including hydraulic jump and rotation," ASME J. Heat Transfer 112:728-735

4. Rahman, M.M., Faghri, A., Hankey, W., 1991, "Computation of turbulent flow in a thin liquid layer of fluid involving a hydraulic jump," J. Fluids Engr. 113 pp. $411-418$

5. Avedisian, C. T. and Zhao, Z, 2000, "The circular hydraulic jump in low gravity," Proc. Roy. Soc. London, 456 pp. 2127-2151

6. Labus, T.L. and DeWitt, K.J., 1978, "Liquid jet impingement normal to a disk in zero gravity," J. Fluids Engr. 100, pp. 204-209

7. Miyasaka, Y.,1974, "On the flow of a viscous free boundary jet on a rotating disk," Bull J. Soc. Mech. Eng. ,17, pp. 1469-1475

8. Rahman, M.M. and Faghri, A.,1992, "Numerical simulation of fluid flow and heat transfer in a thin liquid film over a rotating disk," Int. J. Heat Mass Transfer, 35, pp. $1441-1453$

9. Buyevich, Y.A. and Ustinov, V.A., 1994, "Hydrodynamic conditions of transfer processes through a radial jet spreading over a flat surface," Int. J. Heat Mass Transfer, 37, pp.165-173

10. Rao, A. and Arakeri, J.H., 1998, "Integral analysis applied to radial film flows," Int. J. Heat Mass Transfer , 41, pp. 2757-2767

11. Azuma, T. and Hoshino, T. 1984, The radial flow of a thin liquid film, 1st-4th Reports. Bull. J. Soc. Mech. Eng., 27, pp. 2739-2770

12. Ozar, B., Cetegen, B. M. and Faghri, A., 2003, "Experiments on the flow of a thin liquid film over a horizontal stationary and rotating disk surface," Exp. Fluids, 34, pp. $556-565$

13. Chadhury, Z. H., 1964, “Heat Transfer in a Radial Liquid Jet," J. Fluid Mech., 20, pp. 501-511. 
14. Wang, X. S., Dagan, Z., and Jiji, L. M., 1989, "Heat Transfer Between a Circular Free Impinging Jet and a Solid Surface with Non-Uniform Wall Temperature of Wall Heat Flux: 1: Solution for the Stagnation Region," Int. J. Heat Mass Transfer, 32, pp. 1351-1360.

15. Carper, H. J., and Defenbaugh, D. M., 1978, "Heat Transfer from a Rotating Disk with Liquid Jet Impingement,' Proceedings of 6th Int. Heat Transfer Conference. Toronto, pp. 113-118.

16. Carper, Jr., H. J., Saavedra, J. J., and Suwanprateep, T., 1986, "Liquid Jet Impingement Cooling of a Rotating Disk," ASME J. Heat Transfer, 108, pp. $540-546$.

17. Vader, D. T., Incropera, F. P., and Viskanta, R., 1991, "Local Convective Heat Transfer From a Heated Surface to an Impinging, Planar Jet of Water," Int. J. Heat Mass Transfer, 34, pp. 611-623.

18. Stevens, J., and Webb, B. W., 1991, "Local Heat Transfer Coefficients Under and Axisymmetric, Single-Phase Liquid Jet," ASME J. Heat Transfer, 113, pp. 71-78.

19. Faghri, A., Thomas, S., and Rahman, M. M., 1993, "Conjugate Heat Transfer from a Heated Disk to a Thin Liquid Film Formed by a Controlled Impinging Jet," ASME J. Heat Transfer, 115, pp. 116-123.

20. Aoune, A., and Ramshaw, C., 1999, "Process Intensification: Heat and Mass Transfer Characteristics of Liquid Films on Rotating Discs," Int. J. Heat Mass Transfer, 42, pp. 2543-2556.

21. Nusselt WZ (1916) Die Oberflachenkondensation des Wasserdampfes. Z. Ver Deut. Ing., 60 , pp. 541-546

22. Ozar, B., Cetegen, B. M., Faghri, A., "Experiments on Heat Transfer in a Thin Liquid Film Flowing Over a Rotating Disk," ASME J. Heat Transfer, 126, pp. 184-192

23. Rice, J., Faghri, A., Cetegen, B. M., "Analysis of a free surface film from a controlled liquid impingement jet over a rotating disk including conjugate effects with and without evaporation", Paper No. HT2005-72103, Proc. HT 2005 ASME Summer Heat Transfer Conference, July 17-22, 2005, San Francisco, CA, U.S.A. (also submitted to Int. J. Heat Mass Transfer) 


\section{APPENDIX A}

For determining the thermal entry length for the constant disk surface temperature case, the temperature profile in this region is taken as,

$$
\theta=\left[\left(\frac{z}{\delta_{T}}\right)^{2}-2\left(\frac{z}{\delta_{T}}\right)\right]
$$

Introducing, $\hat{z} \equiv \frac{\tilde{z}}{\tilde{\delta}_{T}}$ and $\beta=\frac{\delta_{T}}{\delta}$, the previously determined velocity profile and the nondimensional temperature profiles become,

$$
\tilde{u}_{r}=3 \frac{\hat{z}}{\tilde{r} \tilde{\delta}_{T}^{2}} \beta^{2} \frac{3}{2} \frac{\hat{z}^{2}}{\tilde{r} \tilde{\delta}_{T}{ }^{3}} \beta^{3} \quad \theta=\hat{z}^{2}-2 \hat{z}
$$

substituting into the integral energy equation and integrating to $\hat{z}=1$, one gets

$$
\beta \frac{d}{d \tilde{r}}\left[\frac{\beta^{2}}{4}-\frac{\beta^{3}}{20}\right]=\frac{2}{P e}\left(\frac{r_{o}}{h_{o}}\right)^{2} \frac{\tilde{r}}{\tilde{\delta}}
$$

since $\beta<1, \beta^{3}<<\beta^{2}$, the equation governing $\beta$ becomes,

$$
\beta^{2} \frac{d \beta}{d \tilde{r}}=\frac{4}{P e}\left(\frac{r_{0}}{h_{0}}\right)^{2} \frac{\tilde{r}}{\tilde{\delta}}
$$

with the condition that $\beta(\tilde{r}=1)=0$. The solution is obtained by direct integration as,

$$
\beta=\left[\frac{12}{P e}\left(\frac{r_{o}}{h_{o}}\right)^{2} \int_{1}^{\tilde{r}} \frac{\tilde{r} d \tilde{r}}{\tilde{\delta}}\right]^{1 / 3}
$$

If we assume that $\tilde{\delta} \approx 1$, then the integral can be evaluated as,

$$
\tilde{r}_{*}=\left[1+\frac{P e}{6}\left(\frac{h_{0}}{r_{0}}\right)^{2}\right]^{1 / 2}
$$

Since $h_{o}<<r_{o}$, then, $\tilde{r}_{*} \approx 1$ or $r_{*} \approx r_{o}$. The smallness of the entry region length is utilized in the heat transfer analysis presented in the main body of the paper. 


\section{APPENDIX B}

Considering the integral momentum equation given by eqn. (9), neglecting the inertial terms on the left hand side allows the direct integration between $\tilde{z}=0$ and $\tilde{z}=\tilde{\delta}$ to yield the velocity profile,

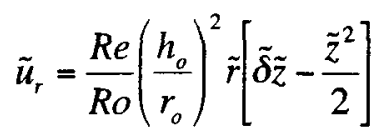

The corresponding film thickness can be obtained upon integration for the total volumetric flow rate of liquid at any radius leading to the expression for $\tilde{\delta}$ given by eqn. (14). Substitution of this velocity profile into the energy integral equation leads to the following differential equation for $a_{0}$

$$
\frac{d a_{0}}{d \tilde{r}}=\frac{1}{\operatorname{Re} \operatorname{Pr}}\left(\frac{r_{o}}{h_{o}}\right)^{2}+\frac{3^{1 / 3} 7}{30}\left(\frac{\operatorname{Ro}}{\operatorname{Re}}\right)^{1 / 3}\left(\frac{r_{o}}{h_{o}}\right)^{2 / 3} \tilde{r}^{-5 / 3}-\frac{2}{3^{2 / 3}}\left(\frac{h_{o}}{r_{o}}\right)^{1 / 3}\left(\frac{\operatorname{Ro}}{\operatorname{Re}}\right)^{1 / 3} a_{o} \tilde{r}^{-8 / 3}
$$

Since $r_{o}>h_{o}$, the last term on the right hand side is small compared to the others. If neglected, eqn. (B2), becomes directly integrable. Applying the condition $a_{o}(\tilde{r}=1)=1 / 3$, we get,

$$
a_{o}=\left(\frac{r_{o}}{h_{o}}\right)^{2} \frac{\tilde{r}-1}{\operatorname{RePr}}-\frac{3^{1 / 3} 7}{20}\left(\frac{r_{o}}{h_{o}}\right)^{2 / 3}\left(\frac{R o}{\operatorname{Re}}\right)^{1 / 3}\left(\tilde{r}^{-2 / 3}-1\right)+\frac{1}{3}
$$




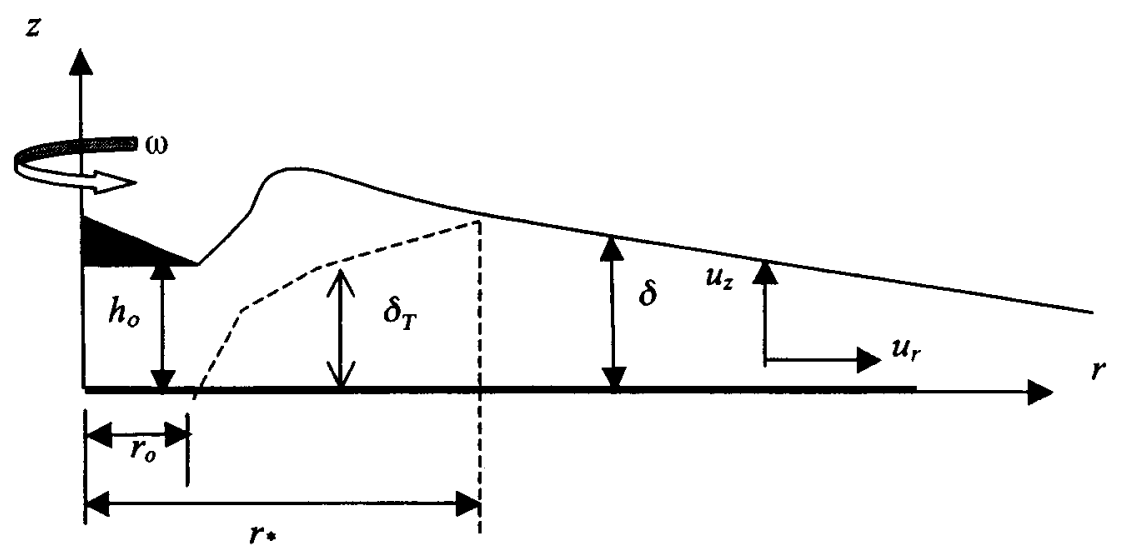

Fig 1: Schematics of the thin film over a rotating disk 

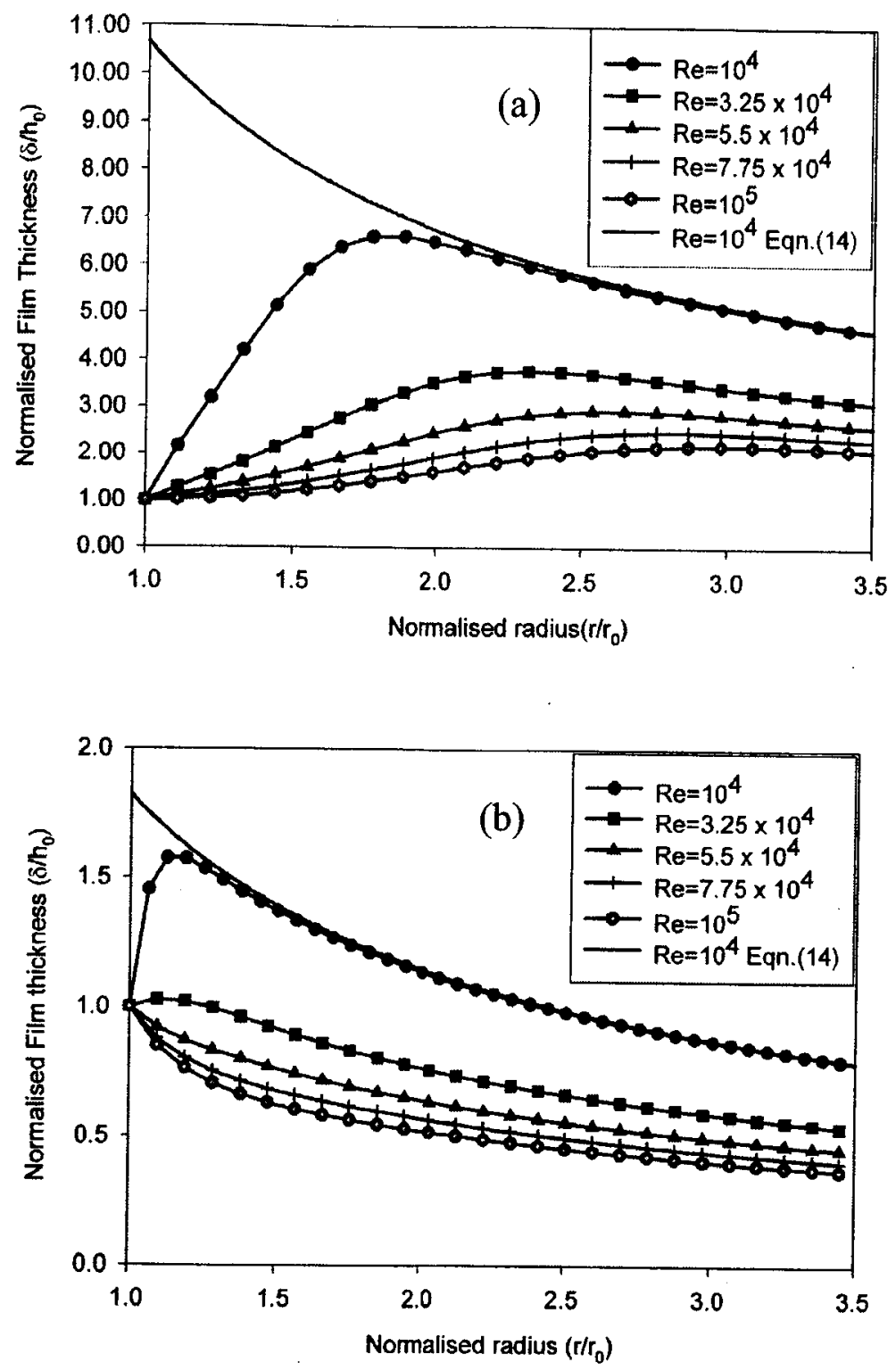

Fig 2: Variation of normalized film thickness as a function of Reynolds number for (a) Ro $=1000$ and (b) $R_{0}=0.5$ 


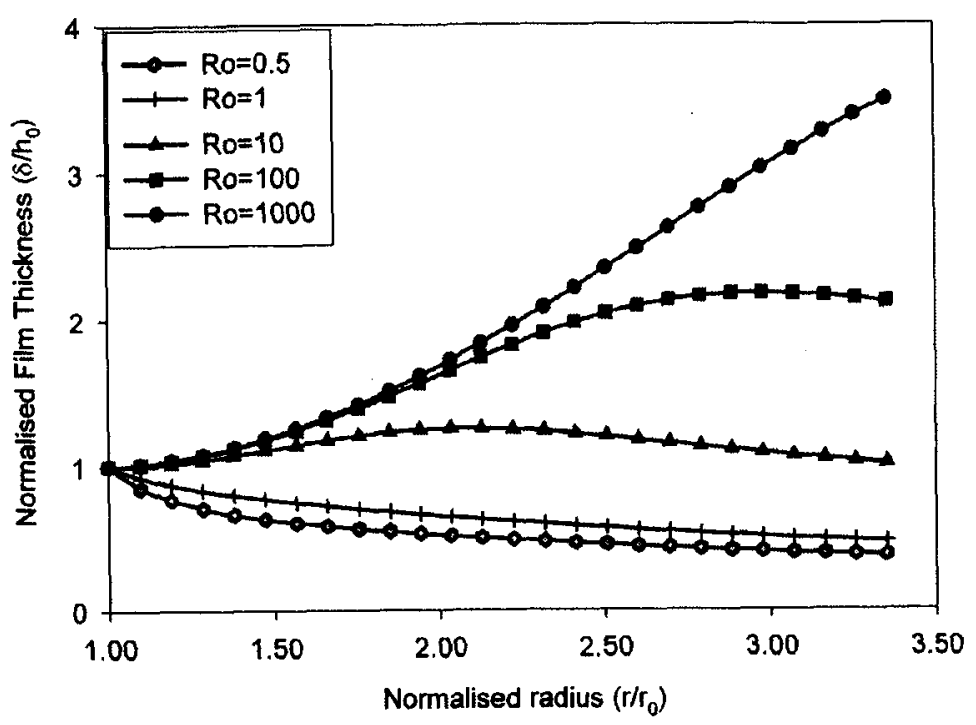

Fig 3: Normalized film thickness variation over the disk surface for different Rossby numbers number for medium flow rate $\operatorname{Re}=10^{5}$

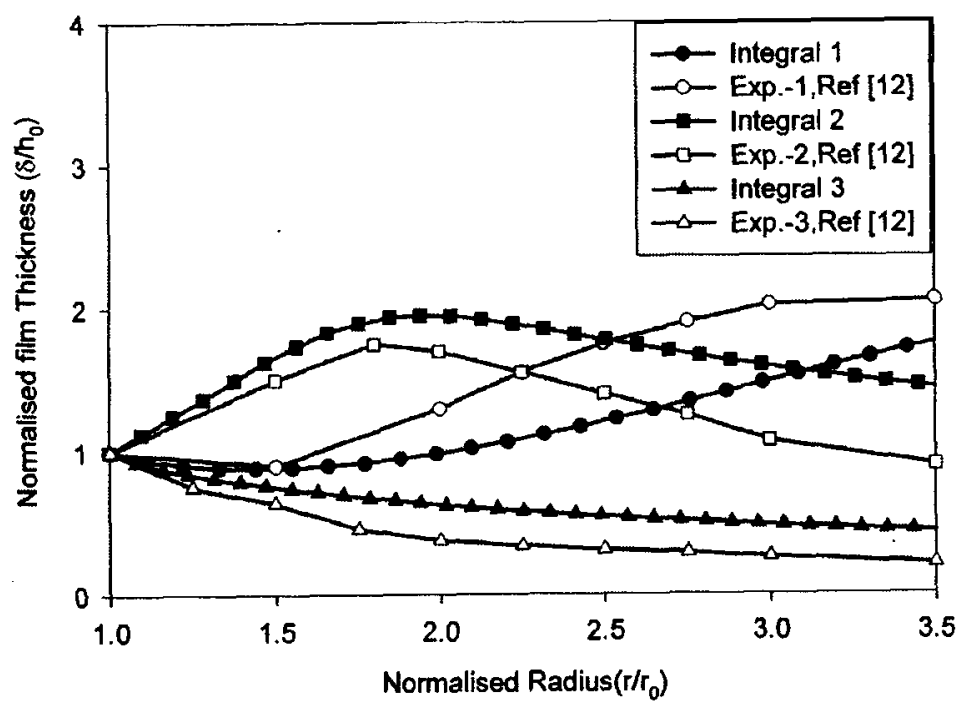

Fig 4: Comparison of film thickness for experimental and theoretical data for several Reynolds number and Rossby numbers. Case 1: $\operatorname{Re}=2.410^{5}, \operatorname{Ro}=361 ;$ Case $2: \operatorname{Re}=4.810^{4}, \operatorname{Ro}=361$; Case 3: $\operatorname{Re}=4.810^{4}, \operatorname{Ro}=0.4$ 

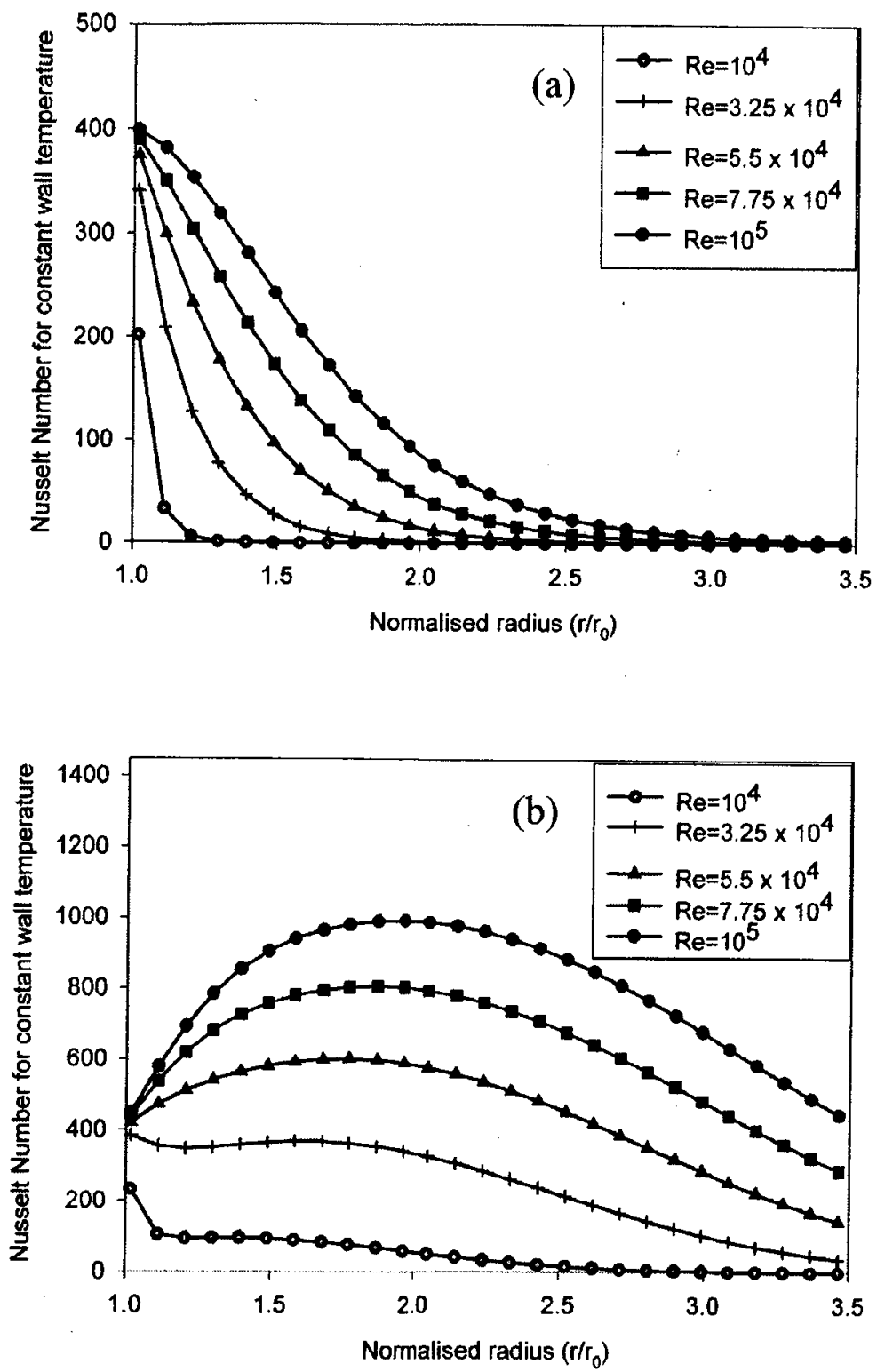

Fig 5: Constant wall temperature Nusselt number variation over the disk surface for different Reynolds numbers for (a) low rotation $\mathrm{Ro}=1000$ and (b) high rotation $\mathrm{Ro}=0.5$ cases. 


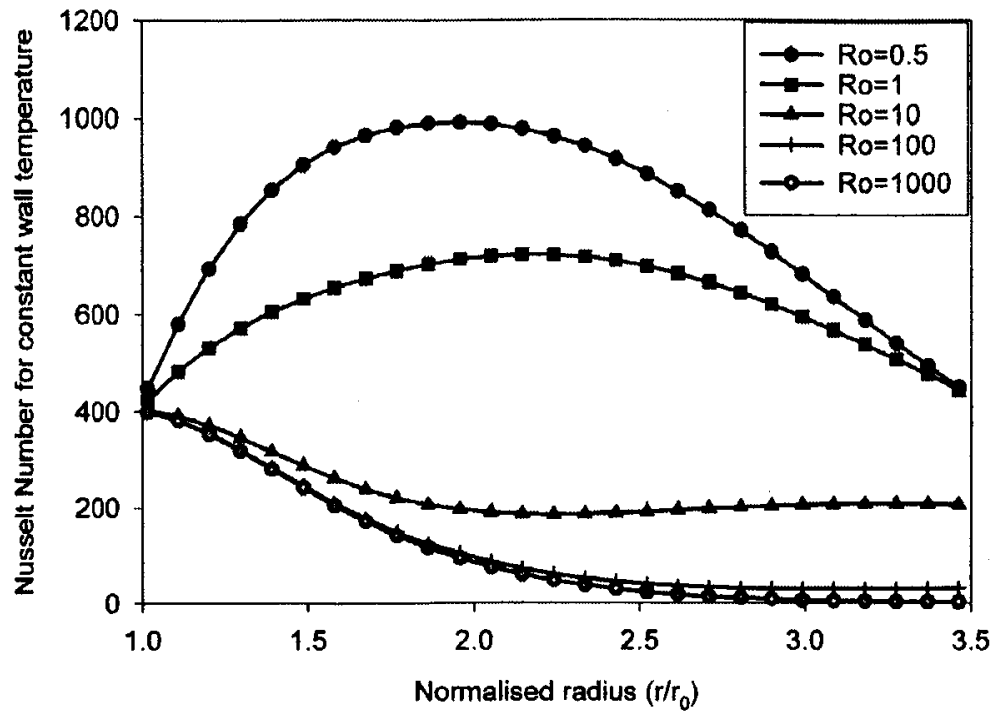

Fig 6: Nusselt number variation for different Rossby numbers for $\mathrm{Re}=10^{5}$ 

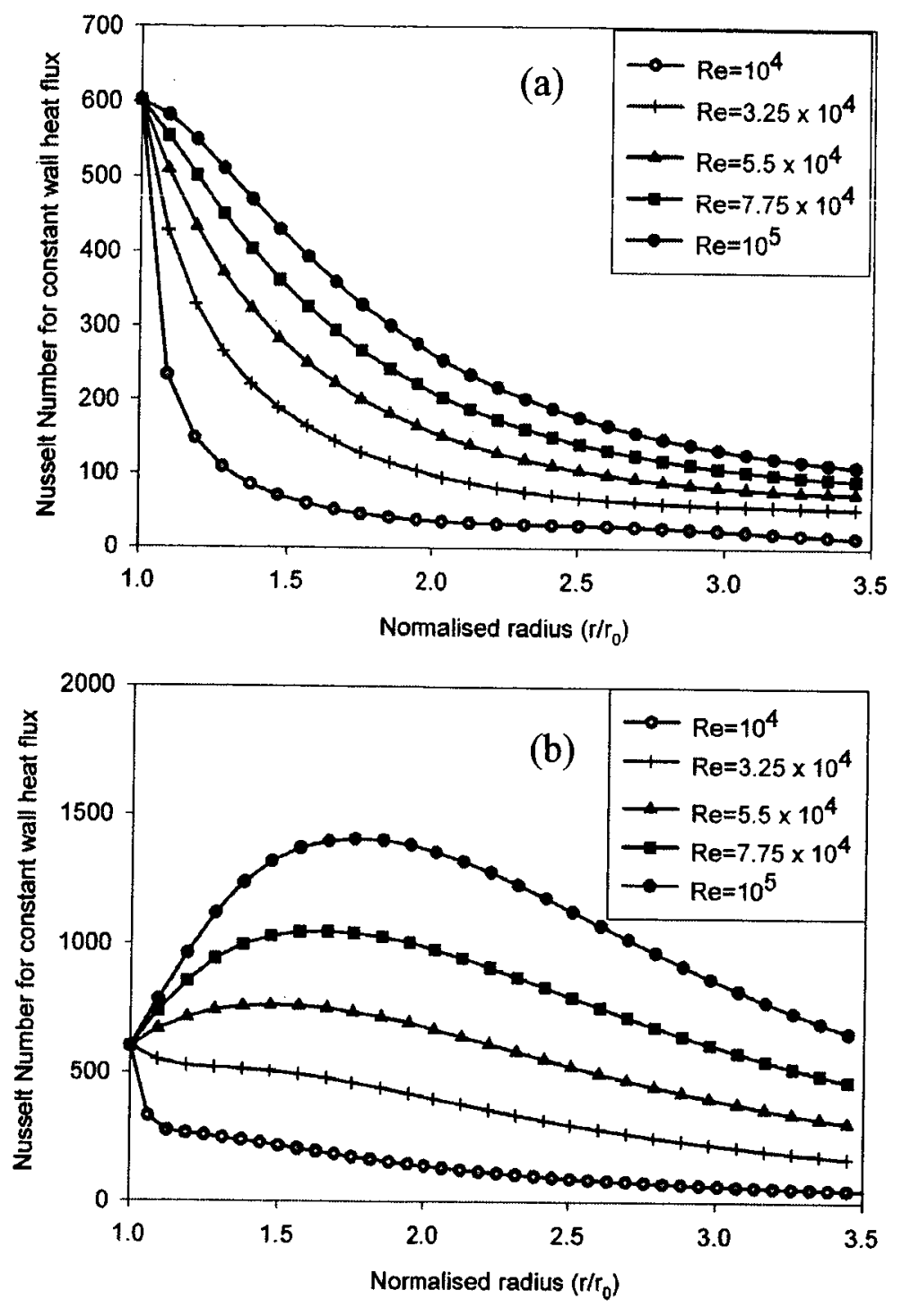

Fig 7: Constant wall heat flux Nusselt number variation over the disk surface for different Reynolds numbers for (a) low rotation Ro $=$ 1000 and (b) high rotation Ro $=0.5$ cases. 


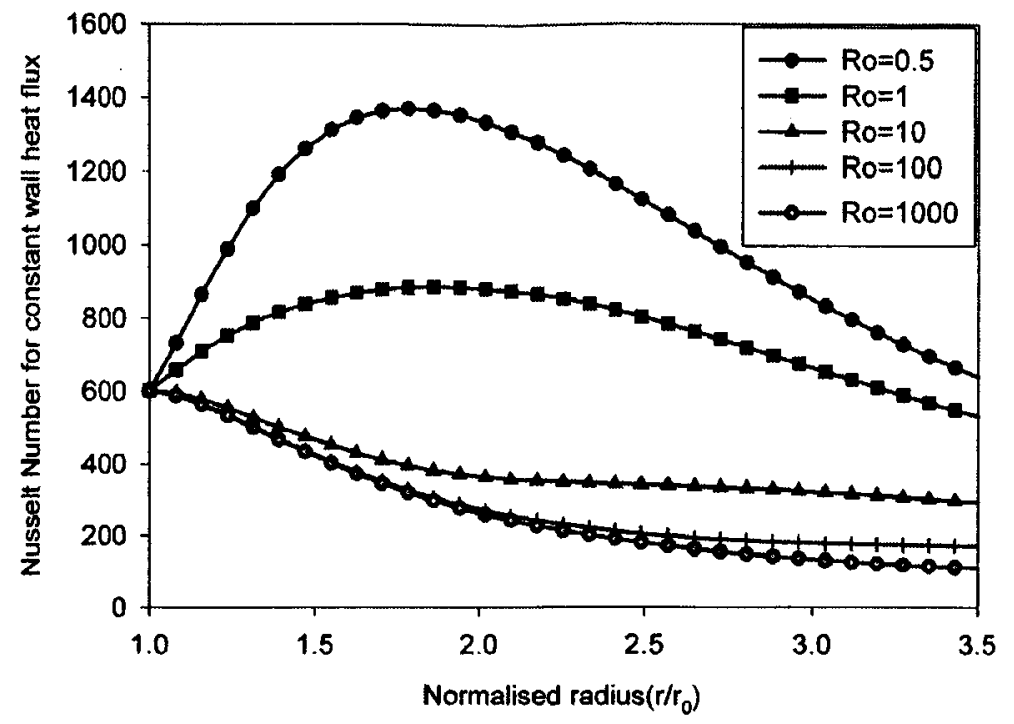

Fig 8: Nusselt number variation for different Rossby numbers for $\operatorname{Re}=10^{5}$ 

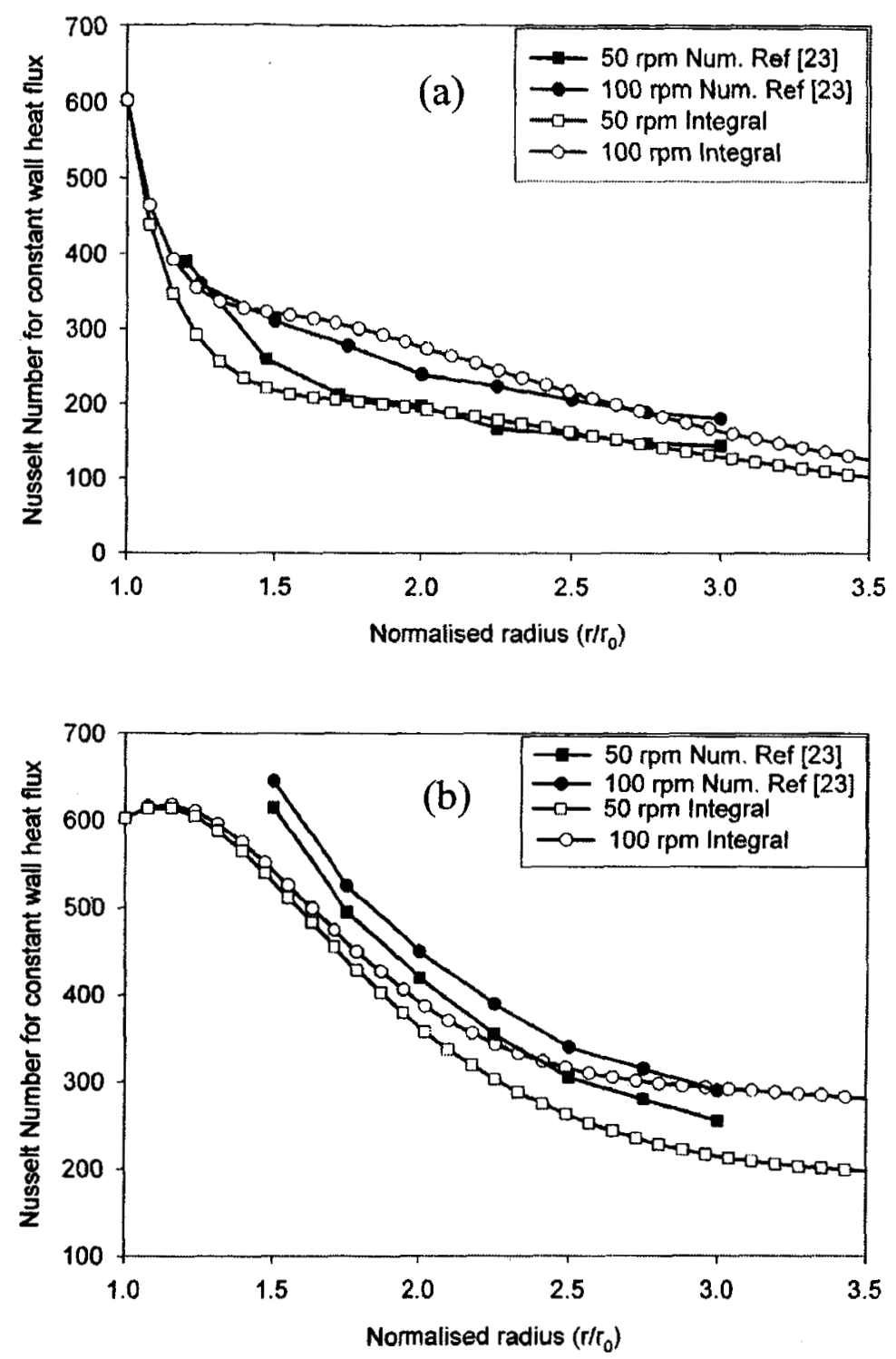

Fig 9: Comparison of calculated Nusselt Number with numerical data of Rice et al [23] for (a) $\operatorname{Re}=2.8410^{4}$ (b) $\operatorname{Re}=1.4210^{5}$ and rotation speeds of $50,100 \mathrm{rpm}$ 

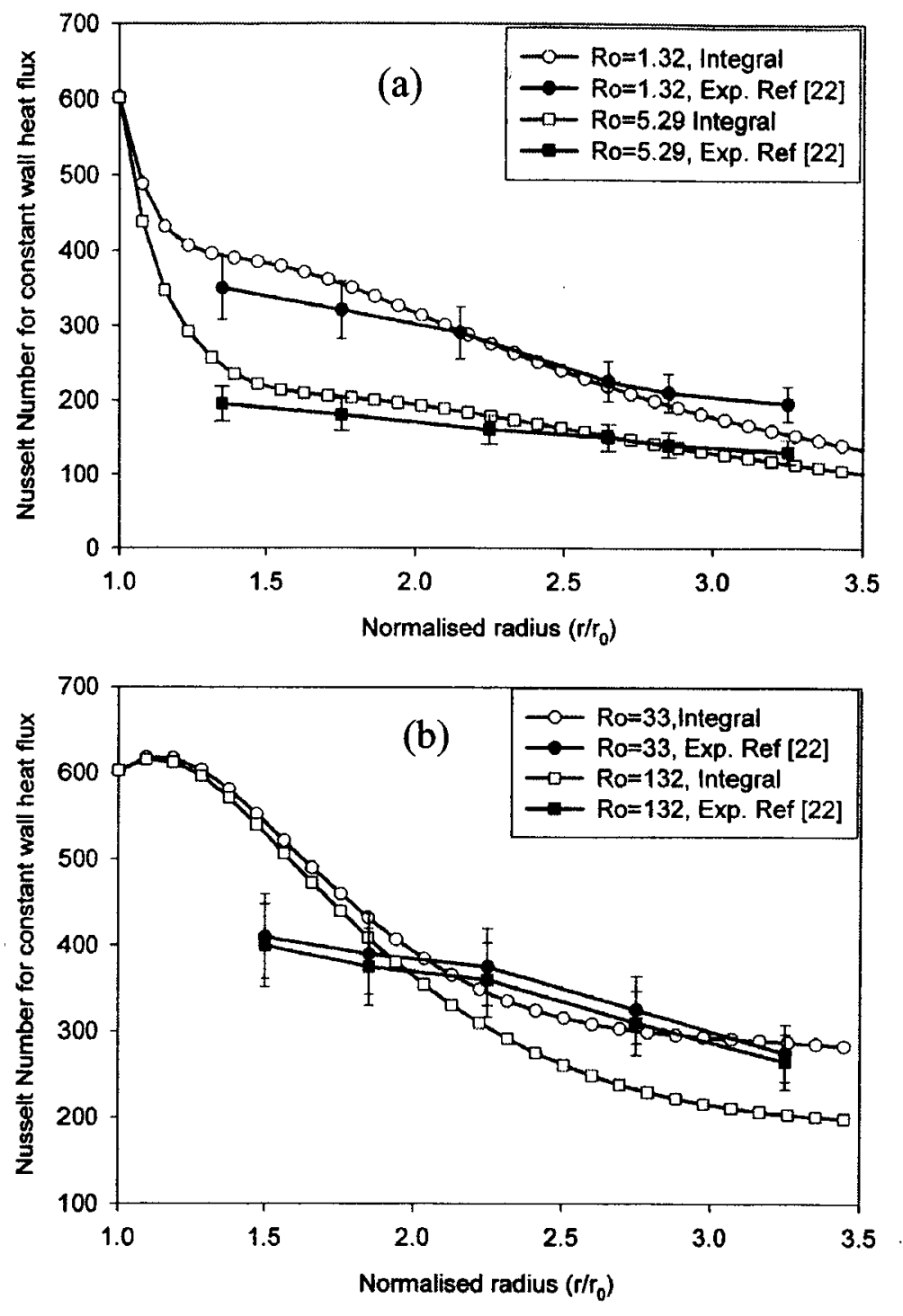

Fig 10: Comparison of Nusselt Number from the integral analysis and the experimental data of Ozar et al [22] for (a) $\mathrm{Re}=2.8510^{4}$ and (b) $R e=1.4210^{5}$ 


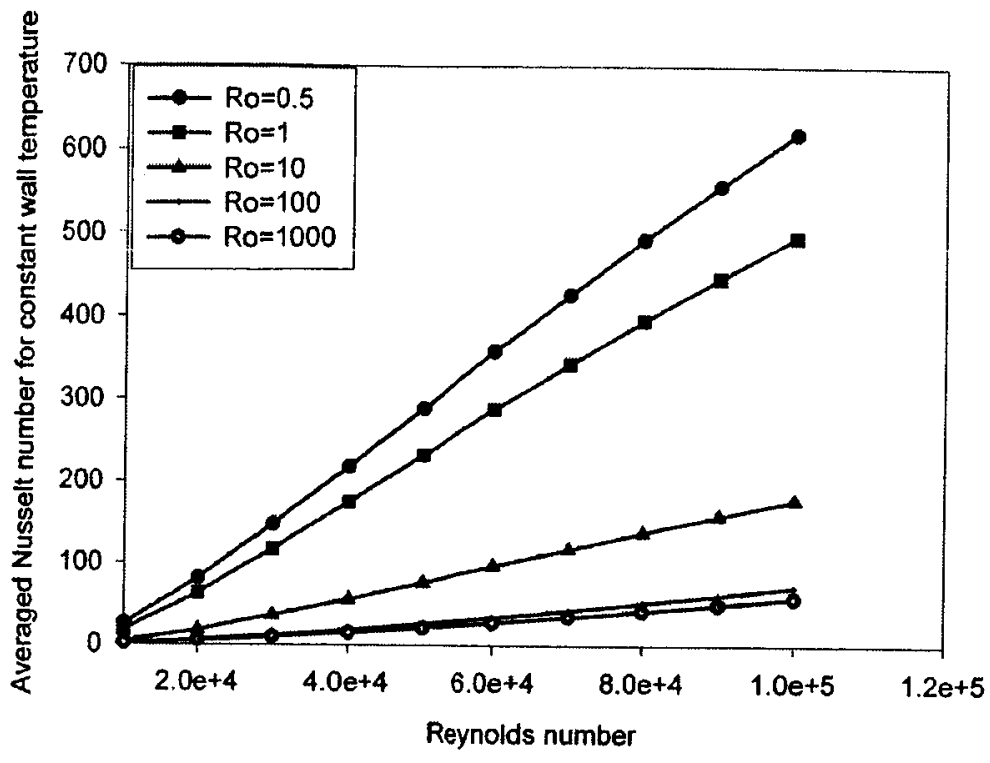

Fig 11: Variation of Average Nusselt Number (based on area) with Reynolds Number for different rotation rates corresponding to the constant wall temperature.

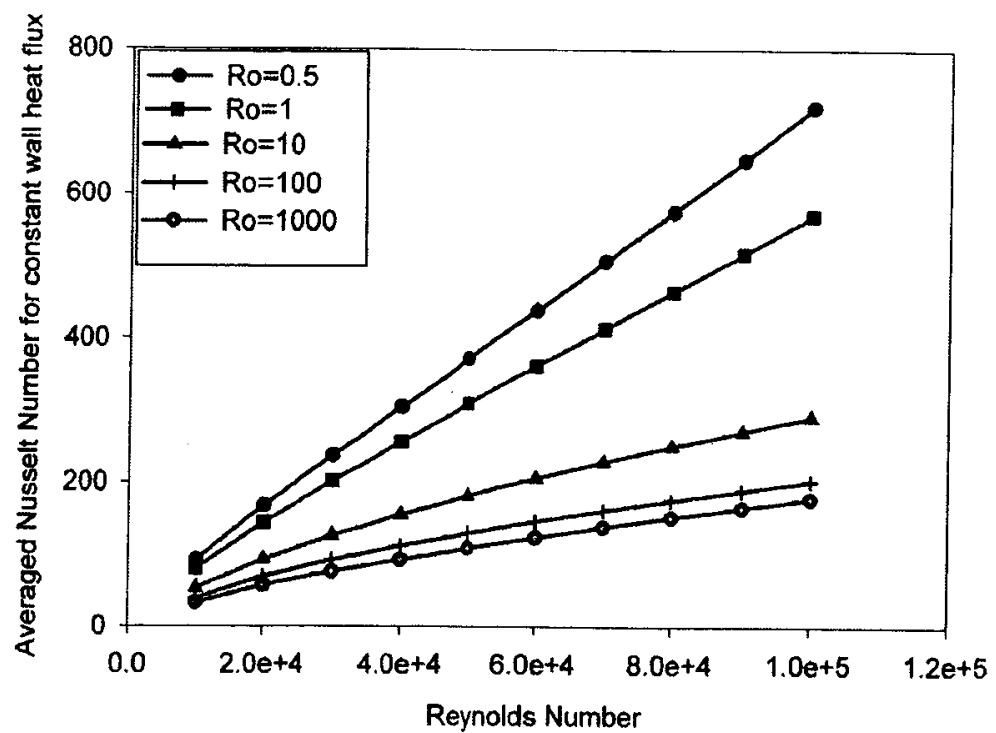

Fig 12: Variation of Average Nusselt Number (based on area) with Reynolds Number for different rotation rates corresponding to the constant wall heat flux case 


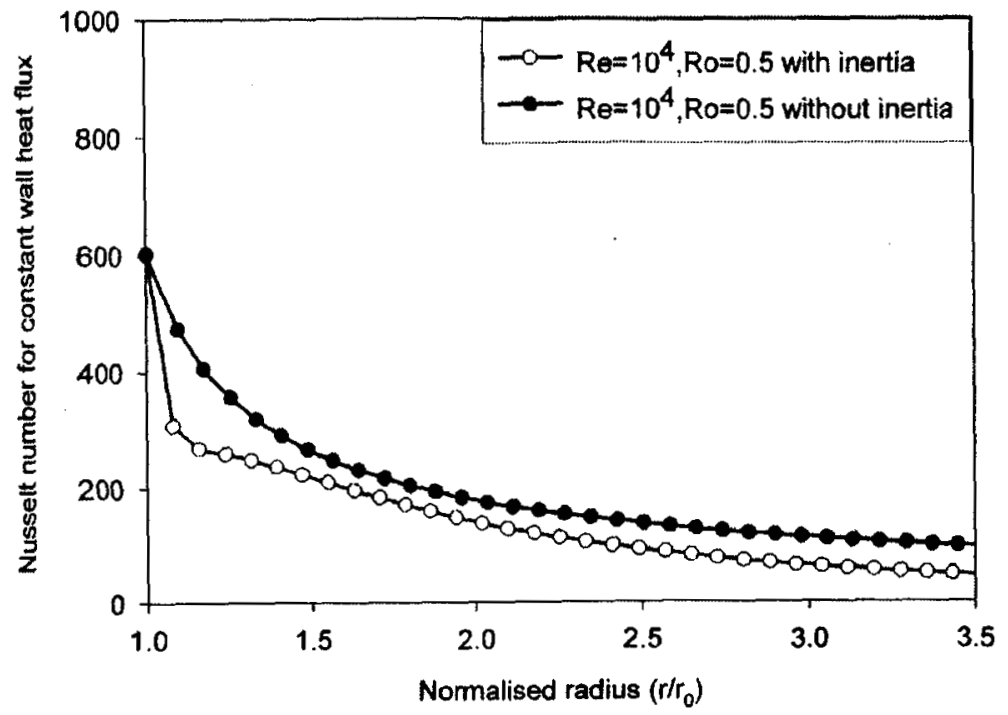

Fig 13: The comparison of the Nusselt number for constant heat flux obtained from full solution and the approximation of negligible inertia 